\title{
Chaotic Vibration of the Wave Equation with Nonlinear Feedback Boundary Control: Progress and Open Questions
}

\author{
Goong Chen ${ }^{1,2}$, Sze-Bi Hsu ${ }^{3}$ and Jianxin Zhou ${ }^{1}$
}

\section{Contents}

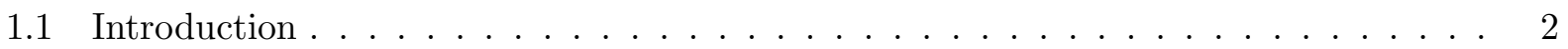

1.2 The linear wave equation with a van der Pol boundary condition . . . . . . . . 4

1.2.1 Chaotic vibration of the classical solution: the case $0<\alpha \leq 1 \ldots 7$

1.2.2 Chaotic vibration when there is hysteresis: the case $\alpha>1 \ldots \ldots$

1.2.3 Memory effects when the displacement term is present in the nonlinear boundary condition . . . . . . . . . . . . . . . . 10

1.2.4 Nonisotropic spatiotemporal chaotic vibration . . . . . . . . . . . . . 13

1.3 Some open questions . . . . . . . . . . . . . . . . 16

\begin{abstract}
The study of chaotic phenomena in partial differential equations is a challenging subject. In this paper, we survey the recent progress in the study of chaotic vibration of the linear wave equation with nonlinear boundary feedback control law. We show that when there is linear energy injection at one end of the boundary and the self-regulating or van der Pol nonlinearity at the other end of the boundary, chaos occurs as a reconciliation between linear instability and nonlinear self-regulation when the parameters enter a certain regime. A list of open problems is also posed.

1. Department of Mathematics, Texas A\&M University, College Station, TX 77843, U.S.A. E-mail: gchen@math.tamu.edu and jzhou@math.tamu.edu,

2. Supported in part by a Texas A\&M University Telecommunication Initiative Task Force (TITF) grant.

3. Department of Mathematics and College of Science, National Tsing Hua University, Hsinchu 30043, Taiwan, R.O.C. Supported in part by a research grant from the National Science Council of R.O.C. E-mail: sbhsu@math.nthu.edu.tw
\end{abstract}




\subsection{Introduction}

The onset of chaotic phenomena in systems governed by nonlinear partial differential equations (PDEs) has fascinated scientists and mathematicians for many centuries. The most famous case in point is the Navier-Stokes equations and related models in fluid dynamics, where the occurrence of turbulence in fluids is well accepted as a chaotic phenomenon. Yet despite the diligence of numerous of the most brilliant minds of mankind, and the huge amount of new knowledge gained through the vastly improved computational and experimental methods and facilities, at present we still have not been able to rigorously prove that turbulence is indeed chaotic in a certain universal mathematical sense.

Nevertheless, rapid advances have been made in nonlinear science that now we do know much more about how and why nonlinear phenomena such as pattern formation, adaptation, self-organization, bifurcation and chaos, etc., happen in a variety of physical systems. So many new results are announced daily that there is no doubt that nonlinear science belongs to the frontiers of science and technology of the $21^{\text {st }}$ Century, offering numerous challenges as well as exciting opportunities.

Look at the mathematics side. Three or four decades ago, the majority of the research publications in the area of nonlinear differential equations still dealt with the existence and uniqueness issues. There seemed to be a mentality set during that period that these were the best qualities a nonlinear system (or any system) should possess, and no other qualities were more worthwhile. Gradually, we saw the shifts of emphases and interests. Bifurcation analysis have become popular, and new methods have been developed to prove multiplicity of solutions of genuinely nonlinear problems (where the linearization method would not lead anywhere). Nowadays, existence and uniqueness are treated mostly as "mundane" issues and few people are interested only in these issues.

Chaos may be viewed as an extreme form of the nonlinear dynamical phenomena. In general, it seems harder to prove the onset of chaos than, e.g., that of bifurcations. For systems of nonlinear ordinary differential equations (ODEs), pioneering work probing the chaotic behavior was done by Lorenz [18] for the Lorenz system and by Cartwright and Littlewood [1] for the forced van der Pol oscillator, among others. A useful mathematical technique to rigorously prove the occurrence of chaos was developed by Melnikov [20] using the Smale Horseshoe; see also [22].

Generalization of the Melnikov method to certain nonlinear PDEs has been made; see [12, 15, 16, 17], e.g. Those PDEs have a Hamiltonian structure available for exploitation. The PDEs (mostly) live on the entire space and, therefore, there are no boundary conditions to worry about.

When boundary conditions are present in a time-dependent nonlinear PDE, analysis becomes very complicated and, to our knowledge, not too much work is available in the literature. But for a special class of PDEs, namely, the wave equation, one can utilize wave reflection on the boundary to analyze or even "manipulate" chaotic behavior. This study actually complements the type of work mentioned in the preceding paragraph $[12,15,16,17]$ where, as we mentioned earlier, boundary conditions are for the most part either not included or not regarded as important in the models.

The historical background of our study came from the boundary stabilization problem of the 
linear wave equation. Let us describe it below. Let

$$
\frac{1}{c^{2}} \frac{\partial^{2} w(x, t)}{\partial t^{2}}-\frac{\partial w(x, t)}{\partial x^{2}}=0, \quad 0<x<1, \quad t>0,
$$

denote the linear PDE modeling either acoustic wave propagation or a vibrating string on the unit interval $(0,1)$, where $c>0$ denotes the speed of wave propagation. At the left-end $x=0$, assume that the boundary condition is fixed:

$$
w(0, t)=0, \quad t>0 .
$$

At the right-end $x=1$, control is placed:

$$
w_{x}(1, t)=u(1, t), \quad t>0 .
$$

The initial conditions are

$$
w(x, 0)=w_{0}(x), \quad w_{t}(x, 0)=w_{1}(x), \quad 0<x<1,
$$

for two given functions $w_{0}$ and $w_{1}$ with sufficient smoothness, satisfying $w(0, t)=0$ for all $t>0$. The energy associated with vibration at time $t$ is

$$
E(t)=\frac{1}{2} \int_{0}^{1}\left[w_{x}^{2}(x, t)+\frac{1}{c^{2}} w_{t}^{2}(x, t)\right] d x .
$$

The objective of the stabilization problem is to find a feedback law for $u(t)$ in (1.3) such that

$$
\lim _{t \rightarrow \infty} E(t)=0 .
$$

A simple choice of the feedback law is the negative velocity feedback:

$$
u(t)=-\alpha w_{t}(1, t), \quad t>0, \quad \alpha>0, \quad \alpha \neq 1 / c,
$$

under the assumption that the velocity $w_{t}(1, t)$ at $x=1$ can be observed and be fedback. Substituting (1.7) into (1.3), we obtain the so-called viscous damping boundary condition

$$
w_{x}(1, t)+\alpha w_{t}(1, t)=0, \quad t>0 .
$$

With this boundary condition, the energy of the system dissipates with time $t$ :

$$
\begin{aligned}
\frac{d}{d t} E(t) & =\int_{0}^{1}\left[w_{x}(x, t) w_{x t}(x, t)+\frac{1}{c^{2}} w_{t}(x, t) w_{t t}(x, t)\right] d x \\
& \quad \text { integration by parts } \Rightarrow) \\
& =\left.w_{x}(x, t) w_{t}(x, t)\right|_{x=0} ^{x=1} \\
& =-\alpha w_{t}^{2}(1, t) \leq 0 .
\end{aligned}
$$

Using the method of characteristics (see Section 1.2), one can further show that the energy decays with an exponential rate:

$$
E(t) \leq K e^{-\mu t} E(0), \quad \text { for some } K>0 \text { independent of }\left(w_{0}, w_{t}\right),
$$

where the exponential rate $e^{-\mu t}$ with $\mu=-\frac{c}{2} \ln \left|\frac{1-\alpha c}{1+\alpha c}\right|>0$ is sharp. Thus, (1.10) is actually a uniform exponential stabilization result, where by "uniform" we mean the decay rate is uniform with respect to any initial condition $\left(w_{0}, w_{1}\right)$ given in (1.4). This uniform stabilization result is also useful in solving the exact controllability problem: 
"For any given sufficiently smooth functions $z_{0}(x)$ and $z_{1}(x)$ on $[0,1]$, satisfying $z_{0}(0)=0$, find a controller $u(t)$ in (1.3) such that at the terminal time $T>0$,

$$
w(T, x)=z_{0}(x), \quad w_{t}(T, x)=z_{1}(x), \quad 0<x<1 .
$$

Using the "controllability via stabilizability" method of Russell [21] for time-reversible distributed parameter systems, one can prove that the exact controllability problem is solvable if $T>0$ is sufficiently large. (The provision that $T$ be sufficiently large cannot be weakened because the wave propagates with a finite speed and it takes a certain amount of time for the boundary control effect to be propagated to the entire interval.)

So the linear feedback boundary condition (1.8) is nice and useful. However, in the design of many servomechanisms, stabilizability or controllability are not issues of any concern. What is really of concern is the safe or robust operation of the servomechanism. One such example is the classical van der Pol equation

$$
\ddot{x}-\left(\alpha-\beta \dot{x}^{2}\right) \dot{x}+k x=0 ; \quad \alpha, \beta>0,
$$

where $x=x(t)$ is proportional to the electric current at time $t$ on a circuit equipped with a van der Pol device. Then the energy at time $t$ is $E(t)=\frac{1}{2}\left(\dot{x}^{2}+k x^{2}\right)$ and

$$
\frac{d}{d t} E(t)=\dot{x}(\ddot{x}+k x)=\dot{x}^{2}\left(\alpha-\beta \dot{x}^{2}\right)
$$

so we have

$$
E^{\prime}(t)\left\{\begin{array}{lll}
\geq 0 & \text { if } & |\dot{x}| \leq(\alpha / \beta)^{1 / 2}, \\
<0 & \text { if } & |\dot{x}|>(\alpha / \beta)^{1 / 2}
\end{array}\right.
$$

which is the desired self-regulation effect, i.e., energy will increase when $|\dot{x}|$ is small which is unfit for operation, and energy will decrease when $|\dot{x}|$ is large in order to prevent electric current surge which may destroy the circuit. (This self-regulating effect is also called self-excitation.) A second version of the van der Pol equation is

$$
\ddot{x}-\left(\alpha-3 \beta x^{2}\right) \dot{x}+k x=0,
$$

which may be regarded as a differentiated version of (1.11), satisfying a regulation effect similar to (1.12). Neither (1.11) nor (1.13) has any chaotic behavior as the solutions tend to limit cycles according to the Poincaré-Bendixon Theorem. However, when a forcing term A cos(cot) is added to the right hand side of (1.11) or (1.13), solutions display chaotic behavior when the parameters $A$ and $\omega$ enter a certain regime $[12,14]$.

What happens when we study the PDE analogue of (1.11) or (1.13) for the wave equation? This is one of the major motivations of our study to be delineated in Section 1.2. In Section 1.3, we provide what we regard as a set of interesting open questions for further research.

\subsection{The linear wave equation with a van der Pol boundary condition}

In this section, we survey four cases of chaos generation or anticontrol by nonlinear feedback boundary control. Consider (1.1), but set the wave speed $c=1$ therein because $c$ is not an essential parameter as far as the mathematical analysis of chaotic vibration is concerned. Thus, we consider

$$
w_{t t}(x, t)-w_{x x}(x, t)=0, \quad 0<x<1, \quad t>0 .
$$


Repeat the two initial conditions in (1.4) here:

$$
w(x, 0)=w_{0}(x), \quad w_{t}(x, 0)=w_{1}(x), \quad 0<x<1 .
$$

At the right-end $x=1$, assume a nonlinear boundary condition

$$
w_{x}(1, t)=\alpha w_{t}(1, t)-\beta w_{t}^{3}(1, t) ; \quad t>0, \quad \alpha, \beta>0 .
$$

At the left-end $x=0$, we have options to choose several types of boundary conditions. Here, let us choose it to be

$$
w_{t}(0, t)=-\eta w_{x}(0, t), \quad t>0 ; \quad \eta>0, \quad \eta \neq 1 .
$$

Remark 1.1. Equation (1.17) says that negative force is fedback to the velocity at $x=0$. An alternate choice would be

$$
w_{x}(0, t)=-\eta w_{t}(0, t), \quad t>0 ; \quad \eta>0, \quad \eta \neq 1,
$$

which says negative velocity is fedback to force.

With (1.16) and (1.17), we have, by (1.9), (1.16) and (1.17),

$$
\begin{aligned}
\frac{d}{d t} E(t) & =\frac{d}{d t} \int_{0}^{1}\left[\frac{1}{2} w_{x}^{2}(x, t)+w_{t}^{2}(x, t)\right] d t \\
& =\eta w_{x}^{2}(0, t)+w_{t}^{2}(1, t)\left[\alpha-\beta w_{t}^{2}(1, t)\right] .
\end{aligned}
$$

The contribution $\eta w_{x}^{2}(0, t)$ above, due to $(1.17)$, is always nonnegative. Thus we see that the effect of (1.17) is to cause energy to increase. For this reason, the boundary condition (1.17) is said to be energy-injecting or energy-pumping. On the other hand, we have

$$
w_{t}^{2}(1, t)\left[\alpha-\beta w_{t}^{2}(1, t)\right]\left\{\begin{array}{lll}
\geq 0 & \text { if } & \left|w_{t}(1, t)\right| \leq(\alpha / \beta)^{1 / 2}, \\
<0 & \text { if } & \left|w_{t}(1, t)\right|>(\alpha / \beta)^{1 / 2}
\end{array}\right.
$$

so the contribution of the boundary condition (1.16) to (1.18) is self-regulating because (1.19) works in exactly the same way as (1.12). Thus, we call (1.16) a van der Pol, self-regulating, or self-excitation, boundary condition. Intuitively speaking, with the boundary condition (1.17) alone (and with the right-end boundary condition (1.16) replaced by a conservative boundary condition such as $w(1, t)=0$ or $w_{x}(1, t)=0$ for all $\left.t>0\right)$ it causes the well-known classical linear instability, namely, the energy grows with an exponential rate:

$$
E(t)=\mathcal{O}\left(e^{k t}\right), \quad k=\frac{1}{2} \ln \left(\left|\frac{1+\eta}{1-\eta}\right|\right)>0 .
$$

However, the self-regulating boundary condition (1.16) can hold the instability (1.20) partly in check by its regulation effect, for a large class of bounded initial states with bounds depending on the parameters $\alpha, \beta$ and $\eta$. When $\alpha, \beta$ and $\eta$ match in a certain regime, chaos happens, which could be viewed as a reconciliation between linear instability and nonlinear self-regulation. Overall, there is a richness of nonlinear phenomena, including: the existence of asymptotically periodic solutions, hysteresis, instability of the type of unbounded growth, and fractal invariant sets. 
A basic approach for the problems under consideration in this section is the method of characteristics. Let $u$ and $v$ be the Riemann invariants of (1.14) defined by

$$
\begin{aligned}
& u(x, t)=\frac{1}{2}\left[w_{x}(x, t)+w_{t}(x, t)\right], \\
& v(x, t)=\frac{1}{2}\left[w_{x}(x, t)-w_{t}(x, t)\right] .
\end{aligned}
$$

Then $u$ and $v$ satisfy a diagonalized first order linear hyperbolic system

$$
\frac{\partial}{\partial t}\left[\begin{array}{l}
u(x, t) \\
v(x, t)
\end{array}\right]=\left[\begin{array}{cc}
1 & 0 \\
0 & -1
\end{array}\right] \frac{\partial}{\partial x}\left[\begin{array}{l}
u(x, t) \\
v(x, t)
\end{array}\right], \quad 0<x<1, \quad t>0,
$$

with initial conditions

$$
\begin{aligned}
& u(x, 0)=u_{0}(x) \equiv \frac{1}{2}\left[w_{0}^{\prime}(x)+w_{1}(x)\right], \\
& v(x, 0)=v_{0}(x) \equiv \frac{1}{2}\left[w_{0}^{\prime}(x)-w_{1}(x)\right],
\end{aligned}
$$

The boundary condition (1.16), after converting to $u$ and $v$ and simplifying, becomes

$$
u(1, t)=F_{\alpha, \beta}(v(1, t)), \quad t>0,
$$

where the relation $u=F_{\alpha, \beta}(v)$ is defined implicitly by

$$
\beta(u-v)^{3}+(1-\alpha)(u-v)+2 v=0 ; \quad \alpha, \beta>0 .
$$

Remark 1.2. For (1.25), we know that

(i) when $0<\alpha \leq 1$, for each $v \in \mathbb{R}$, there exists a unique $u \in \mathbb{R}$;

(ii) when $\alpha>1$, for each $v \in \mathbb{R}$, in general there may exist two or three distinct $u \in \mathbb{R}$ satisfying (1.25). Thus $u=F_{\alpha, \beta}(v)$ is not a function relation.

Case (i) will be treated in Subsection 1.2.1 while case (ii), containing hysteresis, will be treated in Subsection 1.2.2.

The boundary conditions (1.14), by (1.21), becomes

$$
v(0, t)=G_{\eta}(u(0, t)) \equiv \frac{1+\eta}{1-\eta} u(0, t), \quad t>0 .
$$

Equations (1.25) and (1.26) are, respectively, the wave-reflection relations at the right-end $x=1$ and the left-end $x=0$. The reflection of characteristics is depicted in Fig. 2.1.

Assume that $F_{\alpha, \beta}$ is well defined. Then a solution $(u, v)$ of the system (1.22), (1.23), (1.24) and (1.26) can be expressed as follows:

For $0 \leq x \leq 1$ and $t=2 k+\tau$, with $k=0,1,2, \ldots$, and $0 \leq \tau<2$,

$$
\begin{aligned}
& u(x, t)= \begin{cases}(F \circ G)^{k}\left(u_{0}(x+\tau)\right), & \tau \leq 1-x, \\
G^{-1} \circ(G \circ F)^{k+1}\left(v_{0}(2-x-\tau)\right), & 1-x<\tau \leq 2-x, \\
(F \circ G)^{k+1}\left(u_{0}(\tau+x-2)\right), & 2-x<\tau \leq 2 ;\end{cases} \\
& v(x, t)= \begin{cases}(G \circ F)^{k}\left(v_{0}(x-\tau)\right), & \tau \leq x, \\
G \circ(F \circ G)^{k}\left(u_{0}(\tau-x)\right), & x<\tau \leq 1+x, \\
(G \circ F)^{k+1}\left(v_{0}(2+x-\tau)\right), & 1+x<\tau \leq 2,\end{cases}
\end{aligned}
$$


where in the above, $F=F_{\alpha, \beta}$ and $G=G_{\eta}$, and $(G \circ F)^{k}$ represents the $k$-th iterate of the map $G \circ F$. From now on, we often abbreviate $F_{\alpha, \beta}$ and $G_{\eta}$, respectively, as $F$ and $G$, in case no ambiguities will occur. We call the map $G_{\eta} \circ F_{\alpha, \beta}$, naturally, the composite reflection relation. This map $G_{\eta} \circ F_{\alpha, \beta}$ can be regarded as the Poincaré section of the PDE system because we can essentially construct the solution from $G_{\eta} \circ F_{\alpha, \beta}$ using (1.27).

From (1.27), it becomes quite apparent that the solutions $(u(x, t), v(x, t))$ will manifest chaotic behavior when the map $G \circ F$ is chaotic, in the sense of Devaney [11, p. 50], for example. We proceed with the discussion in the following four subsections. The main sources for Subsections 1.2.1-1.2.4 are, in sequential order respectively, [4, 5, 9, 7].

\subsubsection{Chaotic vibration of the classical solution: the case $0<\alpha \leq 1$}

As mentioned in Remark 1.1, when $0<\alpha \leq 1$, for each $v \in \mathbb{R}$ there exists a unique $u \in \mathbb{R}$ such that $u=F_{\alpha, \beta}(v)$. Therefore, the solution $(u, v)$ to (1.22), (1.23), (1.24) and (1.26) is unique. When the initial condition $\left(u_{0}, v_{0}\right)$ is sufficiently smooth satisfying compatibility conditions with the boundary conditions, then $(u, v)$ will also be $C^{1}$-smooth on the spatiotemporal domain.

Let $\alpha$ and $\beta$ be fixed, and let $\eta>0$ be the only parameter that varies. To aid understanding, we include a sample graph of the map $G_{\eta} \circ F_{\alpha, \beta}$, with $\alpha=1 / 2, \beta=1$, and $\eta=0.552$, in Fig. 2.2. We only need to establish that $G_{\eta} \circ F_{\alpha, \beta}$ is chaotic, because $F_{\alpha, \beta} \circ G_{\eta}$ is topologically conjugate to $G_{\eta} \circ F_{\alpha, \beta}$ through

$$
F_{\alpha, \beta} \circ G_{\eta}=G_{\eta}^{-1} \circ\left(G_{\eta} \circ F_{\alpha, \beta}\right) \circ G_{\eta}
$$

and, thus, the iterates $(F \circ G)^{k}$ or $(F \circ G)^{k+1}$ appearing in $(1.27)$ do not need to be treated separately.

We note the following bifurcations: For fixed $\alpha: 0<\alpha \leq 1$ and $\beta>0$, let $\eta \in(0,1)$ be varying.

(1) Period-doubling bifurcation [4, p. 431, Theorem 3.1]

Define

$$
h(v, \eta)=-G_{\eta} \circ F(v)
$$

and let

$$
v_{0}(\eta) \equiv \eta[(1+\eta) / 2][(\alpha+\eta) / \beta]^{1 / 2}
$$

which, for each $\eta$, represents a fixed point of $h$, i.e.,

$$
h\left(v_{0}(\eta), \eta\right)=v_{0}(\eta)
$$

Then the algebraic equation

$$
\frac{1}{2}\left(\frac{1+\alpha \eta}{3 \beta \eta}\right)^{1 / 2}\left[\frac{1+(3-2 \alpha) \eta}{3 \eta}\right]=\frac{1+\eta}{2}\left(\frac{\alpha+\eta}{\beta}\right)^{1 / 2}
$$

has a unique solution $\eta=\eta_{0}: 0<\eta_{0} \leq \boldsymbol{\eta}_{H}$, where

$$
\boldsymbol{\eta}_{H} \equiv\left(1-\frac{1+\alpha}{3 \sqrt{3}}\right) /\left(1+\frac{1+\alpha}{3 \sqrt{3}}\right)
$$

satisfying

$$
\left.\frac{\partial}{\partial v} h_{1}(v, \eta)\right|_{\substack{v=v_{0}\left(\eta_{0}\right) \\ \eta=\eta_{0}}}=-1
$$


which is the primary necessary condition for period-doubling bifurcation to happen, at $v=v_{0}\left(\eta_{0}\right), \eta=\eta_{0}$. Furthermore, the other "accessory" conditions are also satisfied, and the bifurcationed period-2 solutions are attracting.

Consequently, there is a period-doubling route to chaos, as illustrated in the orbit diagram in Fig. 2.3.

(2) Homoclinic orbits [4, pp. 436-437, Theorem 4.1]

Let $\boldsymbol{\eta}_{H}$ be given by (1.28). If

$$
\eta_{H} \leq \eta<1,
$$

then $M \geq I_{1}$ (cf. Fig. 2.2) and, consequently, the repelling fixed point 0 of $G_{\eta} \circ F$ has homoclinic orbits. Furthermore, if $\eta=\boldsymbol{\eta}_{H}$, then there are degenerate homoclinic orbits (and, thus, homoclinic bifurcations [11, p. 125]).

When $M>I_{2}$; cf. Fig. 2.2 , then $\left[-I_{2}, I_{2}\right] \times\left[-I_{2}, I_{2}\right]$ is no longer an invariant square for the map $G \circ F$. What happens is exactly similar to the case of the quadratic map $f_{\mu}(x)=\mu x(1-x)$, for $0 \leq x \leq 1$, when $\mu>4$ because part of the graph of $f_{\mu}$ will protrude above the unit square. See Fig. 2.4. It is easy to see that now the map $G \circ F$ has a Cantor-like fractal invariant set $\Lambda$ on the interval $\left[-I_{2}, I_{2}\right]$, where $\Lambda=\bigcap_{j=1}^{\infty}(G \circ F)^{k}\left(\left[-I_{2}, I_{2}\right]\right)$. All the other points outside $\Lambda$ are eventually mapped to $\pm \infty$ as the number of iterations increases.

We furnish a PDE example below.

Example 1.1 ([4, p. 435, Example 3.3]). Consider (1.22), (1.23), (1.24) and (1.26), where we choose

$$
\begin{gathered}
\alpha=0.5, \beta=1, \eta=0.525 \approx \boldsymbol{\eta}_{H}, \text { satisfying }(1.31), \\
w_{0}(x)=0.2 \sin \left(\frac{\pi}{2} x\right), \quad w_{1}(x)=0.2 \sin (\pi x), \quad x \in[0,1] .
\end{gathered}
$$

Two spatiotemporal profiles of $u$ and $v$ are plotted, respectively, in Figs. 2.5 and 2.6. Their rugged outlooks manifest chaotic vibration.

\section{Miscellaneous remarks}

(1) In this subsection, we have illustrated only the case $0<\eta<1$. When $\eta>1$, the results are similar. See [4].

(2) With the nonlinear boundary condition (1.16), we can only establish that $u$ and $v$ are chaotic. From this, we can then show that $w_{x}$ and $w_{t}$, i.e., the gradient of $w$, are also chaotic by a natural topological conjugacy, see [3, Section 5]. However, $w$ itself is not chaotic because $w$ is the time integral of $w_{t}$, which smooths out the oscillatory behavior of $w_{t}$.

In order to have chaotic vibration of $w$, one must use a differentiated boundary condition; see [4, Section 6]. This is actually an analog of (1.13).

(3) When the initial data $\left(u_{0}, v_{0}\right)$ takes values outside the invariant square $\left[-I_{2}, I_{2}\right] \times\left[I_{2}, I_{2}\right]$, then part of $u$ and $v$ will diverge to $\pm \infty$ as $t \rightarrow \infty$. This behavior belongs to classical unbounded instability. 


\subsubsection{Chaotic vibration when there is hysteresis: the case $\alpha>1$}

When $\alpha>1$, the relation $u=F_{\alpha, \beta}(v)$ is no longer single-valued. (The notation $u \in F_{\alpha, \beta}(v)$ would be more appropriate.) For each value of $v \in \mathbb{R}$, there may exist up to three $u \in \mathbb{R}$ satisfying (1.25). We plot a graph of $u=G_{\eta} \circ F_{\alpha, \beta}(v)$ in Fig. 2.7. Throughout this subsection, we require $0<\eta<1$.

The multi-valued relation $u=G_{\eta} \circ F_{\alpha, \beta}(v)$ consists of three piecewise single-valued branches, $H_{1}, H_{2}$ and $H_{3}$ (where $H$ stands for "hysteresis"), to be defined below. First, let $v^{*} \equiv$ $\frac{\alpha-1}{3}\left[\frac{\alpha-1}{3 \beta}\right]^{1 / 2}$. Note that if $v \notin\left[-v^{*}, v^{*}\right]$, then $F_{\alpha, \beta}$ is actually single-valued: it consists of a continuous branch $F_{\alpha, \beta}^{(1)}$, defined for $v<-v^{*}$ and a second branch $F_{\alpha, \beta}^{(3)}$ defined for $v>v^{*}$. Now, let $\widetilde{F}_{\alpha, \beta}^{(1)}$ be the maximal single-valued continuous extension of the continuous function $F_{\alpha, \beta}^{(1)}$, and let $\widetilde{F}_{\alpha, \beta}^{(3)}$ similarly be the maximal single-valued continuous extension of the function $F_{\alpha, \beta}^{(3)}$ such that

$$
F_{\alpha, \beta}^{(i)}(v) \in F_{\alpha, \beta}(v), \quad \text { for } \quad i=1 \text { or } 3 .
$$

Then we see that $\widetilde{F}_{\alpha, \beta}^{(1)}$ is defined for $v \in\left(-\infty, v^{*}\right]$, and $\widetilde{F}_{\alpha, \beta}^{(3)}$ is defined for $\left[-v^{*}, \infty\right)$. We define $\widetilde{F}_{\alpha, \beta}^{(2)}$ to be

$$
\begin{aligned}
& u=\widetilde{F}_{\alpha, \beta}^{(2)}(v) \text { if } v \in\left(-v^{*}, v^{*}\right) \text { and } \widetilde{F}_{\alpha, \beta}^{(2)}(v) \in F_{\alpha, \beta}(v) \\
& \text { but } \widetilde{F}_{\alpha, \beta}^{(2)}(v) \neq \widetilde{F}_{\alpha, \beta}^{(i)}(v) \text { for } i=1,3 .
\end{aligned}
$$

Now we define

$$
\begin{array}{llll}
u=H_{1}(v) \equiv G_{\eta} \circ \widetilde{F}_{\alpha, \beta}^{(1)}(v) & \text { if } & v \in\left(-\infty, v^{*}\right], \\
u=H_{2}(v) \equiv G_{\eta} \circ \widetilde{F}_{\alpha, \beta}^{(2)}(v) & \text { if } & v \in\left(-v^{*}, v^{*}\right), \\
u=H_{3}(v) \equiv G_{\eta} \circ \widetilde{F}_{\alpha, \beta}^{(3)}(v) & \text { if } & v \in\left[-v^{*}, \infty\right) .
\end{array}
$$

These three branches are also illustrated in Fig. 2.7. We know that when two branches have overlapping domains, such as $H_{1}$ and $H_{2}$ overlap over $\left(-\infty, v^{*}\right] \cap\left(-v^{*}, v^{*}\right)$, or $H_{2}$ and $H_{3}$ do over $\left(-v^{*}, v^{*}\right) \cap\left[-v^{*}, \infty\right)$, there is a selection rule in favor of the branch with more stability, in this case $H_{1}$ is selected over $H_{2}$, and $H_{3}$ over $H_{2}$ as well, because the slopes on the $H_{1}$ and $H_{3}$ branches have smaller magnitude than the counterpart on $H_{2}$. We now define the hysteresis iterates [5, p. 451, Def. 2.1] as follows: for $u_{0} \in \mathbb{R}, u_{k}=H^{k}\left(u_{0}\right)$ is given inductively according to:

(i) For $k=1, u_{1} \equiv H\left(u_{0}\right)$, where

$$
u_{1}= \begin{cases}H_{1}\left(u_{0}\right), & \text { if } u_{0}<-v^{*} \\ H_{2}\left(u_{0}\right), & \text { if } u_{0} \in\left[-v^{*}, v^{*}\right] \\ H_{3}\left(u_{0}\right), & \text { if } u_{0}>v^{*}\end{cases}
$$

(ii) For $k=2, u_{2} \equiv H^{2}\left(u_{0}\right)$, where

$$
u_{2}= \begin{cases}H_{1}\left(u_{1}\right), & \text { if either } u_{1} \leq-v^{*} \text { or if } u_{0}<-v^{*} \text { and } u_{1} \in\left[-v^{*}, v^{*}\right], \\ H_{2}\left(u_{1}\right), & \text { if } u_{0} \in\left[-v^{*}, v^{*}\right], u_{1} \in\left(-v^{*}, v^{*}\right), \\ H_{3}\left(u_{1}\right), & \text { if either } u_{1} \geq v^{*} \text { or if } u_{0}>v^{*} \text { and } u_{1} \in\left[-v^{*}, v^{*}\right],\end{cases}
$$

for $u_{1}=H\left(u_{0}\right)$. 
(iii) Assume that $u_{j}=H^{j}\left(u_{0}\right)$ are defined for $j=1,2, \ldots, k, k \geq 2$. We define $u_{k+1}=$ $H^{k+1}\left(u_{0}\right)$ by

$$
u_{k+1}= \begin{cases}H_{1}\left(u_{k}\right), & \text { if either } u_{k} \leq-v^{*} \text { or if } u_{k-1}<-v^{*} \text { and } u_{k} \in\left[-v^{*}, v^{*}\right], \\ H_{2}\left(u_{k}\right), & \text { if } u_{0} \in\left[-v^{*}, v^{*}\right], u_{1}, u_{2}, \ldots, u_{k} \in\left(-v^{*}, v^{*}\right), \\ H_{3}\left(u_{k}\right), & \text { if either } u_{k} \geq u^{*} \text { or if } u_{k-1}>v^{*} \text { and } u_{k} \in\left[-v^{*}, v^{*}\right] .\end{cases}
$$

When $\eta=0$, i.e., no energy injection, then it is known that when $\alpha$ increases (with $\beta$ held fixed), the hysteresis iteration $u=H^{*}(v)$ has periodic orbits with larger and larger periods, but no chaos [5, Section 3]. However, if $\eta>0$, then under the following sufficient conditions, chaos occurs.

Theorem 1.1 ([5, p. 467, Theorem 4.1]). Let $0<\eta<1, \alpha>1$ and $\beta>0$ such that

$$
\widetilde{m} \equiv \frac{1+\eta}{1-\eta} \frac{1+\alpha}{3}\left[\frac{1+\alpha}{3 \beta}\right]^{1 / 2} \leq \frac{1+\eta}{2 \eta}\left[\frac{1+\alpha \eta}{\beta \eta}\right]^{1 / 2} .
$$

Define $\theta_{0}=-v^{*} \equiv \frac{\alpha-1}{3}\left[\frac{\alpha-1}{3 \beta}\right]^{1 / 2}$, and let

$$
\theta_{j+1}=H_{3}^{-1}\left(\theta_{j}\right), \quad j=0,1,2, \ldots .
$$

If for some $j \geq 1$, we have $\tilde{\theta}_{j-1}<v^{*}$, and $\tilde{\theta}_{j}, \tilde{\theta}_{j+1}, \tilde{\theta}_{j+2} \in\left[v^{*}, \widetilde{m}\right]$, then the hysteresis map $u=H(v)$ is chaotic on the interval $[-\widetilde{m}, \widetilde{m}]$.

Remark 1.3. The proof of Theorem 1.1 basically shows that property (1.36) (see the next subsection) is true. The hysteresis map $H$ in Theorem 1.1 is essentially a piecewise continuous map, though not single-valued. Thus, useful ideas from Keener [13] can be adopted as well as adapted for our purpose here, and elsewhere [3]. But now we can use a more unified approach based on the exponential growth of total variations as developed in $[8,10]$ by proving the property (1.36) instead.

Example 1.2 ([5, p. 468, Example 4.1]). Choose $\eta=1 / 2, \alpha=2, \beta=1$, and

$$
\left.\begin{array}{l}
u_{0}(x)=u_{0}(0)+x\left[u_{0}(1)-u_{0}(0)\right], \\
v_{0}(x)=u_{0}(x)^{2}+b u_{0}(x)+c,
\end{array}\right\} \quad 0 \leq x \leq 1
$$

with $u_{0}(0)=0.5, u_{0}(1)=H\left(v_{0}(1)\right), b=-0.32830, c=1.4145$ and so, $v_{0}(0)=1.5, v_{0}(1)=1.5$. Then the initial data $u_{0}$ and $v_{0}$ satisfy the compatibility conditions, and

$$
\begin{aligned}
& v^{*}=0.1925, \theta_{0}=-0.1925, \theta_{1}=1.6461, \theta_{2}=2.4408, \theta_{3}=2.7710, \theta_{4}=2.9065, \\
& \ldots, \lim _{j \rightarrow \infty} \theta_{3}=3, \widetilde{m}=3,
\end{aligned}
$$

and the conditions of Theorem 1.1 are satisfied. The solution $(u, v)$ displays chaotic vibration, as can be seen in Fig. 2.8 .

\subsubsection{Memory effects when the displacement term is present in the nonlin- ear boundary condition}

Throughout this subsection, we assume that $0<\alpha<1$ and $\eta>0, \eta \neq 1$.

The nonlinear boundary condition (1.16) does not contain the displacement term $w(1, t)$. However, such a term can naturally occur due to symmetry and reduction of dimensionality. 
Example 1.3 ([9, pp. 966-967]). The wave equation in 3D

$$
\frac{\partial^{2}}{\partial x_{1}^{2}} W(\vec{x}, t)+\frac{\partial^{2}}{\partial x_{2}^{2}} W(\vec{x}, t)+\frac{\partial^{2}}{\partial x_{3}^{2}} W(\vec{x}, t)-\frac{1}{c^{2}} \frac{\partial^{2} W(\vec{x}, t)}{\partial t^{2}}=0, \quad t>0,
$$

where $\vec{x}=\left(x_{1}, x_{2}, x_{3}\right) \in \Omega=\left\{\vec{x} \in \mathbb{R}^{3}|a<| \vec{x} \mid<b\right\}$, for some $a>0, b>0$, i.e., $\Omega$ is the 3D spherical-annular domain with radius $a$ of the inner shell and radius $b$ of the outer shell. The boundary conditions are

$$
\begin{aligned}
& \frac{\partial W(\vec{x}, t)}{\partial n}=\alpha W_{t}(\vec{x}, t)-\beta W_{t}^{3}(\vec{x}, t)-k_{1} W(\vec{x}, t), \quad|\vec{x}|=a, \quad t>0 ; \quad k_{1} \geq 0, \\
& \frac{\partial W(\vec{x}, t)}{\partial n}=\frac{1}{\eta} W_{t}(\vec{x}, t)-k_{2} W(\vec{x}, t), \quad|\vec{x}|=b, \quad t>0 ; \quad \eta>0, \quad k_{2} \geq 0,
\end{aligned}
$$

where $n$ is the unit outward normal at $\partial \Omega$, the boundary of $\Omega$. Assume that the initial conditions are radially symmetric:

$$
\left.\begin{array}{l}
W(\vec{x}, 0)=W_{0}(|\vec{x}|), \\
W_{t}(\vec{x}, 0)=W_{1}(|\vec{x}|)
\end{array}\right\} \quad \vec{x} \in \Omega,
$$

for some sufficiently smooth functions $W_{0}$ and $W_{1}$ defined on $\mathbb{R}$. We can utilize this radial symmetry to effect a reduction of dimensionality by setting

$$
W(\vec{x}, t)=\frac{w(r, t)}{r}, \quad r=|\vec{x}| .
$$

After some manipulation and simplifying assumptions, we obtain a 1D wave equation (1.14), along with (1.15) and (1.17). However, the boundary condition (1.16) now becomes

$$
w_{x}(1, t)=\alpha w_{t}(1, t)-\beta w_{t}^{3}(1, t)-\gamma w(1, t), \quad t>0
$$

for some $\gamma>0$. Note that since $\gamma>0$, the term $\gamma w(1, t)$ in (1.34) cannot be eliminated.

On the boundary $x=1, t>0$, we have

$$
w(1, t)=\int_{0}^{t} w_{t}(1, \tau) d \tau-w(0, t) .
$$

Therefore, the wave-reflection condition (1.25) at the right-end $x=1$ is now modified to be a nonlinear boundary integral equation:

"For given $v(\tau), 0 \leq \tau<1$, and $a_{0} \in \mathbb{R}$, find $u(\tau), 0 \leq \tau<t$ such that

$$
\beta X^{3}(t)+(1-\alpha) X(t)+\gamma\left[\int_{0}^{t} X(\tau) d \tau+a_{0}\right]+2 v(t)=0, \quad t>0,
$$

where $X(t) \equiv u(t)-v(t) . "$

It is not difficult to prove that the nonlinear integral equation (1.35) has a unique solution $u$ when $v$ is sufficiently smooth, say $v$ is $C^{0}$. But the difficulty here is that the integral term

$$
\gamma \int_{0}^{t} v(\tau) d \tau, \text { as part of } \gamma \int_{0}^{t} X(\tau) d \tau=\gamma \int_{0}^{t}[u(\tau)-v(\tau)] d \tau \text { in (1.35), }
$$

now has a memory effect. The Poincaré section of the PDE is no longer an interval map $G_{\eta} \circ F_{\alpha, \beta}$ which we relied so heavily in Subsections 1.2.1 and 1.2.2. The problem is no longer reducible to a 1-dimensional map. It is a genuine infinite dimensional problem. 
For an infinite-dimensional problem, there is first the question of what we mean by chaos in such a system. Here we take an intuitive view that for a dynamical system

$$
\left\{\begin{array}{l}
\frac{d}{d t} x(t)=f(x(t)), \quad t>0 \\
x(0)=x_{0} \in H
\end{array}\right.
$$

where $H$ is a certain function space over the spatial interval $I \subset \mathbb{R}$, we say that the solution $x(\cdot)$ of $(1.36)$ is chaotic if

$$
V_{I}(x(t)) \geq K e^{\mu t}, \text { for some } K, \mu>0, \text { for all } t>0
$$

where $V_{I}(f)$ is the total variation of $f$ on the spatial interval $I$ defined by

$$
\begin{gathered}
V_{I}(f)=\sup _{\mathcal{P}}\left\{\sum_{j=0}^{n-1}\left|f\left(x_{j+1}\right)-f\left(x_{j}\right)\right| \mid\left\{x_{0}, x_{1}, \ldots, x_{n}\right\} \in \mathcal{P},\right. \\
\left.x_{0}<x_{1}<\cdots<x_{n}, x_{j} \in I, j=0,1, \ldots, n\right\}
\end{gathered}
$$

and $\mathcal{P}$ is the set of all partitions $\left\{x_{0}, x_{1}, \ldots, x_{n}\right\}$ of the interval $I$. Thus, (1.37) says that the total variation in time of the solution $x(\cdot)$ grows exponentially. This view and approach is developed in $[8,10]$, motivated by the theorems in [10] that for interval maps, the exponential growth of iterates of total variations is equivalent to the fact that the interval map has a homoclinic orbit, and is thus chaotic.

A key idea in establishing the property (1.37) for our PDE system under study here in this subsection is to exploit the fact that the map $G_{\eta} \circ F_{\alpha, \beta}$ has, in addition to the invariant square $\left[-I_{2}, I_{2}\right] \times\left[-I_{2}, I_{2}\right]$ as indicated in Fig. 2.2, when $M<I_{2}$, also two smaller invariant rectangles contained within, such as Fig. 2.9 indicates visually. This leaves us with some leeway (called the extra margin property in [9]) to treat (1.35) as a (very) small perturbation term so that the solution stays within the large invariant square $\left[-I_{2}, I_{2}\right] \times\left[-I_{2}, I_{2}\right]$. But the restriction is that $\gamma$ must be quite small. We have proved the following.

Theorem 1.2 ([9, pp. 978-979, Theorem 4.1]). Consider (1.22), (1.23), (1.26) and (1.35). Let $w_{0}$ and $w_{1}$ in (1.23) be sufficiently smooth and be compatible with the boundary conditions (1.17) and (1.34) such that $u_{0}=(1 / 2)\left(w_{0}^{\prime}+w_{1}\right)$ and $v_{0}=(1 / 2)\left(w_{0}^{\prime}-w_{1}\right)$ satisfy

$$
\left|v_{0}(x)\right| \leq M_{1}, \quad\left|u_{0}(x)\right| \leq M_{2}, \quad x \in[0,1]
$$

where

$$
\begin{aligned}
M_{1} & \equiv \text { local maximum of } G_{\eta} \circ F_{\alpha, \beta}=M \quad \text { (cf. caption of Fig. 2.2) } \\
& =\left|\frac{1+\eta}{1-\eta}\right| \frac{1+\alpha}{3} \sqrt{\frac{1+\alpha}{3 \beta}}, \\
M_{2} & \equiv \text { local maximum of } F_{\alpha, \beta} \circ G_{\eta} \\
& =\frac{1+\alpha}{3} \sqrt{\frac{1+\alpha}{3 \beta}} .
\end{aligned}
$$

Let $\eta$ satisfy either

$$
0<\left(1-\frac{1+\alpha}{3 \sqrt{3}}\right)\left(1+\frac{1+\alpha}{3 \sqrt{3}}\right)^{-1}<\boldsymbol{\eta}_{0}<1
$$


or

$$
1<\bar{\eta}_{0}<\eta<\left(1-\frac{1+\alpha}{3 \sqrt{3}}\right)^{-1}\left(1+\frac{1+\alpha}{3 \sqrt{3}}\right),
$$

where $\boldsymbol{\eta}_{0}: 0<\boldsymbol{\eta}_{0}<1$ and $\bar{\eta}_{0}: 1<\bar{\eta}_{0}<\infty$ are the unique solution of, respectively, the following equations

$$
\begin{aligned}
& \frac{1+\boldsymbol{\eta}_{0}}{1-\boldsymbol{\eta}_{0}} \frac{1+\alpha}{3}\left(\frac{1+\alpha}{3 \beta}\right)^{1 / 2}=\frac{1+\boldsymbol{\eta}_{0}}{2 \boldsymbol{\eta}_{0}}\left(\frac{1+\alpha \boldsymbol{\eta}_{0}}{\beta \boldsymbol{\eta}_{0}}\right)^{1 / 2}, \\
& \frac{\bar{\eta}_{0}+1}{\bar{\eta}_{0}-1} \frac{1+\alpha}{3}\left(\frac{1+\alpha}{3 \beta}\right)^{1 / 2}=\frac{1+\bar{\eta}_{0}}{2}\left(\frac{\alpha+\bar{\eta}_{0}}{\beta}\right)^{1 / 2} .
\end{aligned}
$$

Assume that $\gamma>0$ is sufficiently small, and that

$$
\text { Range } v_{0} \supseteq\left[-\delta, M_{1}-\delta\right], \quad \text { Range } u_{0} \supseteq\left[-\delta, M_{2}-\delta\right],
$$

for some small $\delta>0$ depending only on $\alpha, \beta, \gamma$ and $\eta$. Then we have

$$
\left.\begin{array}{l}
V_{[0,1]}(u(\cdot, t)) \geq K e^{\mu t} \rightarrow \infty, \\
V_{[0,1]}(v(\cdot, t)) \geq K e^{\mu t} \rightarrow \infty,
\end{array}\right\} \quad \text { as } \quad t \rightarrow \infty,
$$

for some $\mu>\ln 2$, where $V_{[0,1]}$ denotes the total variation on the $x$-interval $[0,1]$.

Snapshots of $u$ and $v$ for some "generic" example are offered in Fig. 2.10.

\subsubsection{Nonisotropic spatiotemporal chaotic vibration}

The chaotic vibrations studied in previous subsections are isotropic in space and time because the governing equation (1.14) is invariant with respect to the change of variables $x \leftrightarrow t$. (In addition, (1.14) is invariant under $x \leftrightarrow-t$.) A somewhat different equation, described by the PDE

$$
w_{x x}(x, t)-\nu w_{x t}(x, t)-w_{t t}(x, t)=0, \quad 0<x<1, \quad t>0 ; \quad \nu>0
$$

contains a special feature that the two families of characteristics propagate with different speeds and, thus, provide a simple model for the analysis of nonisotropic spatiotemporal chaotic vibration.

The time rate of change of energy corresponding to (1.39), subject to boundary conditions

$$
\begin{aligned}
& w_{x}(0, t)=0, \quad t>0, \\
& w_{x}(1, t)=\alpha w_{t}(1, t)-\beta w_{t}^{3}(1, t), \quad t>0 ; \quad \alpha, \beta>0,
\end{aligned}
$$

is then found to be

$$
\begin{aligned}
\frac{d}{d t} E(t) \equiv & \frac{d}{d t}\left[\frac{1}{2} \int_{0}^{1}\left(w_{x}^{2}=w_{t}^{2}\right) d x\right] \\
& (\text { integration by parts } \Rightarrow) \\
= & T_{1}+T_{2},
\end{aligned}
$$

where

$$
T_{1} \equiv \frac{\nu}{2} w_{t}^{2}(0, t), \quad T_{2} \equiv w_{t}^{2}(1, t)\left[\left(\alpha-\frac{\nu}{2}\right)-\beta w_{t}^{2}(1, t)\right] .
$$

The positivity or negativity of $T_{1}$ and $T_{2}$ signifies the following: 
(i) $T_{1} \geq 0$ if $\nu>0$, i.e. there is energy injection into the system indirectly through the term $-\nu w_{x t}$ in (1.39). This $T_{1}$ term would have disappeared if the homogeneous Dirichlet condition $w(0, t)=0$ were imposed at $x=0$ in lieu of the Neumann condition (1.40).

(ii) $T_{2}$ is "regulating" if $\alpha-(\nu / 2)>0$, i.e.

$$
\begin{aligned}
& T_{2} \geq 0 \quad \text { if } \quad\left|w_{t}(1, t)\right| \leq \sqrt{\frac{\alpha-\frac{\nu}{2}}{\beta}} \\
& T_{2}<0 \quad \text { if } \quad\left|w_{t}(1, t)\right|>\sqrt{\frac{\alpha-\frac{\nu}{2}}{\beta}} .
\end{aligned}
$$

Thus energy is increasing if velocity is small, and decreasing if velocity is large.

(iii) $T_{2}$ is dissipative, i.e. $T_{2} \leq 0$, if $\alpha-(\nu / 2) \leq 0$.

Again, we hope that the imbalance between energy injection and the self-regulation effect may lead to chaos.

To study (1.39), we again use the method of characteristics by setting

$$
\begin{aligned}
& u=\frac{1}{\rho_{1}(\nu)+\rho_{2}(\nu)}\left[\rho_{2}(\nu) w_{x}+w_{t}\right], \\
& v=\frac{1}{\rho_{1}(\nu)+\rho_{2}(\nu)}\left[\rho_{1}(\nu) w_{x}-w_{t}\right],
\end{aligned}
$$

where

$$
\rho_{1}(\nu) \equiv\left[-\nu+\left(4+\nu^{2}\right)^{1 / 2}\right] / 2, \quad \rho_{2}(\nu)=\left[\nu+\left(4+\nu^{2}\right)^{1 / 2}\right] / 2 .
$$

We obtain a diagonalized linear symmetric first order hyperbolic system

$$
\frac{\partial}{\partial t}\left[\begin{array}{l}
u(x, t) \\
v(x, t)
\end{array}\right]=\left[\begin{array}{cc}
\rho_{1}(\nu) & 0 \\
0 & -\rho_{2}(\nu)
\end{array}\right] \frac{\partial}{\partial x}\left[\begin{array}{l}
u(x, t) \\
v(x, t)
\end{array}\right], \quad 0<x<t, \quad t>0 .
$$

Physically, the above says that one wave travels to the right with speed $1 / \rho_{1}(\nu)$, while another wave travels to the left with speed $1 / \rho_{2}(\nu)$. A complete cycle of vibration takes $\rho_{1}(\nu)+\rho_{2}(\nu)$ time units. The boundary condition (1.40) gives the reflection relation

$$
v(0, t)=-u(0, t) \equiv G(u(0, t)), \quad t>0,
$$

at the left-end $x=0$, while (1.41) gives

$$
u+v=\alpha\left(\rho_{1} u-\rho_{1} v\right)-\beta\left(\rho_{1} u-\rho_{2} v\right)^{3} ; \quad \rho_{i}=\rho_{i}(\nu) \text { for } i=1,2,
$$

or

$$
\beta X^{3}+\left(\rho_{2}-\alpha\right) X+\left(\rho_{2}^{2}+1\right) v=0 ; \quad X \equiv \rho_{1} u-\rho_{2} v .
$$

In order to have a unique real solution $u$ for a given $v$ from the cubic equation, from now on we require that

$$
\rho_{2}(\nu)-\alpha=\frac{\sqrt{4+\nu^{2}}+\nu}{2}-\alpha \geq 0 .
$$

For each given $v$, then the real solution $u$ of (1.45) is expressed as

$$
u=F_{\nu}(v),
$$


where $\nu>0$ satisfying (1.46) is regarded as the varying parameter, while $\alpha, \beta>0$ are assumed to be held fixed. The unique solution of (1.43)-(1.45), with initial conditions

$$
u(x, 0)=u_{0}(x), \quad v(x, 0)=v_{0}(x), \quad 0<x<1,
$$

can now be expressed explicitly as follows: for $0<x<1$ and for $t=k\left(\rho_{1}+\rho_{2}\right)+\tau, k=$ $0,1,2, \ldots, 0 \leq \tau<\rho_{1}+\rho_{2}$,

$$
\begin{aligned}
& u(x, t)= \begin{cases}\left(F_{\nu} \circ G\right)^{k}\left(u_{0}\left(x+\rho_{1} \tau\right)\right), & \tau \leq \rho_{2}(1-x), \\
F_{\nu} \circ\left(G \circ F_{\nu}\right)^{k}\left(v_{0}\left(1+\rho_{2}^{2}-\rho_{2}^{2}\left(x+\rho_{1} \tau\right)\right),\right. & \rho_{2}(1-x)<\tau \leq \rho_{2}\left(1+\rho_{1}^{2}-x\right), \\
\left(F_{\nu} \circ G\right)^{k+1}\left(u_{0}\left(x+\rho_{1} \tau-1-\rho_{1}^{2}\right)\right), & \rho_{2}\left(1+\rho_{1}^{2}-x\right)<\tau<\rho_{1}+\rho_{2},\end{cases} \\
& v(x, t)= \begin{cases}\left(G \circ F_{\nu}\right)^{k}\left(v_{0}\left(x-\rho_{2} \tau\right)\right), & \tau \leq \rho_{1} x \\
G \circ\left(F_{\nu} \circ G\right)^{k}\left(u_{0}\left(-\rho_{1}^{2}\left(x-\rho_{2} \tau\right)\right)\right), & \rho_{1} x<\tau \leq \rho_{1}\left(x+\rho_{2}^{2}\right), \\
\left(F_{\nu} \circ G\right)^{k+1}\left(v_{0}\left(x-\rho_{2} \tau+1+\rho_{2}^{2}\right)\right), & \rho_{1}\left(x+\rho_{2}^{2}\right)<\tau<\rho_{1}+\rho_{2} .\end{cases}
\end{aligned}
$$

Again, as in (1.27), we see that $G \circ F_{\nu}$ forms a natural Poincaré section for the given PDE system. Chaotic vibration occurs if the map $G \circ F_{\nu}$ is chaotic.

One can analyze that as $\nu$ increases, the map $G \circ F_{\nu}$ has a period-doubling cascade similar to what we have in (1.28)-(1.30) in Subsection 1.2.2. Also, $G \circ F_{\nu}$ has homoclinic orbits and a Cantor-like fractal invariant set when $\nu$ enters a certain regime. For details, see [7].

To conclude, we provide the following example and graphics.

Example 1.4 ([7, pp. 552-554, Example 6.2]). Choose $\alpha=0.5, \beta=1$ and $\nu=3.33$. For these parameters it is known [7] that the map $G \circ F_{\nu}$ is chaotic.

We have

$$
\rho_{1}(\nu)=0.277, \quad \rho_{2}(\nu)=3.61 .
$$

A complete cycle of vibration takes $\rho_{1}+\rho_{2} \approx 3.88$ time units.

For initial conditions, we choose

$$
v_{0}(x) \equiv 0, \quad 0 \leq x \leq 1,
$$

and a $C^{2}$-spline for $u_{0}$ :

$$
\begin{aligned}
& u_{0}(x)=\frac{1}{12} \begin{cases}\frac{\left(x-x_{1}\right)^{3}}{h^{3}}, & x_{1} \leq x \leq x_{2}, \\
1+\frac{3\left(x-x_{2}\right)}{h}+\frac{3\left(x-x_{2}\right)^{2}}{h^{2}}-\frac{3\left(x-x_{2}\right)^{3}}{h^{3}}, & x_{2} \leq x \leq x_{3}, \\
1-\frac{3\left(x-x_{4}\right)}{h}+\frac{3\left(x-x_{4}\right)^{3}}{h^{2}}+\frac{3\left(x-x_{4}\right)^{3}}{h^{3}}, & x_{3} \leq x \leq x_{4}, \\
\frac{\left(x_{5}-x\right)^{3}}{h^{3}}, & x_{4} \leq x \leq x_{5}, \\
0, & \text { elsewhere, }\end{cases} \\
& h=\frac{1}{6}, x_{j}=\frac{j}{6}, \quad j=1,2,3,4,5 .
\end{aligned}
$$

The profiles of $u$ and $v$, as well as the gradient $w_{x}, w_{t}$, are plotted in Figs 2.10 and 2.11. The reader may observe sharp "randomness" in every direction of space and time. Thus, nonisotropic chaotic vibration shows strong mixing of waves. 


\subsection{Some open questions}

We pose a few open questions relevant to the topics discussed in Section 1.2. In our opinion, these are "workable" problems whose resolution will significantly enhance our understanding of chaos in PDEs.

\section{(Q1) Chaotic vibration in 3D}

Example 1.3 in Subsection 1.2.3 shows a 3D problem whose solution has chaotic behavior in the radial variable, as we have successfully reduced the problem to $1 \mathrm{D}$ in terms of the variable $r=|x|=\sqrt{x_{1}^{2}+x_{2}^{2}+x_{3}^{2}}$ (which is rewritten as $x$ in (1.14)). What if the initial conditions in (1.32) contain a small perturbation such as

$$
W(\vec{x}, 0)=W_{0}(|\vec{x}|)+\varepsilon f(\vec{x}), \quad W_{t}(\vec{x}, 0)=W_{1}(|\vec{x}|),
$$

where $f(\vec{x})$ is not a function of $|\vec{x}|$ only?

There is little trouble in believing that chaotic vibration will occur when $\alpha, \beta, \gamma$ and $\eta$ satisfy the assumptions in Theorem 1.2. This will be genuine $3 D$ chaotic vibration because $W(\vec{x}, t)$ depends not only on $|\vec{x}|$ but also on the spherical angular variables $\theta$ and $\phi$ as well.

Can we establish a rigorous proof for this?

\section{(Q2) Chaotic vibration of the wave equation on a 2D annular domain with radial symmetry}

Consider Example 1.3 again, but in 2D, i.e.,

$$
\Omega=\left\{\vec{x} \in \mathbb{R}^{2}|a<| \vec{x} \mid<b\right\} .
$$

Also assume (1.32), so we have radial symmetry. But the wave equation in 2D,

$$
\frac{\partial^{2} W(\vec{x}, t)}{\partial x_{1}^{2}}+\frac{\partial^{2} W(\vec{x}, t)}{\partial x_{2}^{2}}-\frac{1}{c^{2}} \frac{\partial^{2} W(\vec{x}, t)}{\partial t^{2}}=0
$$

is no longer reducible to the form (1.14) because the Huygen's principle does not apply to 2D. The reduced form we can reach is

$$
\frac{\partial^{2} w(r, t)}{\partial r^{2}}+\frac{1}{r} \frac{\partial w(r, t)}{\partial r}-\frac{1}{c^{2}} \frac{\partial^{2} w(r, t)}{\partial t^{2}}=0, \quad r=|\vec{x}|, \quad a<r<b .
$$

For this equation, can we study its chaotic vibrations such as those discussed in Section 1.2?

\section{(Q3) The 1D linear Klein-Gordon equation}

The equation is

$$
\frac{1}{c^{2}} w_{t t}(x, t)-w_{x x}(x, t)+k^{2} w(x, t)=0, \quad 0<x<1, \quad t>0, \quad k^{2}>0 .
$$

The dispersion term $k^{2} w$ above causes significant technical difficulty in the analysis of its behavior when boundary conditions contain nonlinearities.

Can we develop effective methods and devise nonlinear feedback boundary conditions to determine if the system behaves chaotically? 


\section{(Q4) van der Pol nonlinearity distributed over the $x$-span}

We have assumed the van der Pol nonlinearity to live on the boundary $x=1$ such as (1.16). Instead, we may also consider the van der Pol nonlinearity to be distributed

$$
w_{t t}(x, t)+\left[-\alpha w_{t}(x, t)+\beta w_{t}^{3}(x, t)\right]-w_{x x}(x, t)=0, \quad 0<x<1, \quad t>0, \quad \alpha, \beta>0,
$$

and set

$$
w(1, t)=0, \quad t>0
$$

Then we have

$$
\begin{aligned}
\frac{d}{d t} E(t) & =\frac{d}{d t}\left[\frac{1}{2} \int_{0}^{1}\left(w_{x}^{2}+w_{t}^{2}\right) d x\right] \\
& =\eta w_{x}^{2}(0, t)+\int_{0}^{1} w_{t}^{2}\left(\alpha-\beta w_{t}^{2}\right) d x
\end{aligned}
$$

So again we see that the bracketed terms in (1.47) have a self-regulation effect.

Numerical experiments have shown that when $\eta$ enters a certain regime (while $\alpha$ and $\beta$ are held fixed), chaotic vibration occurs.

Can this be rigorously proved?

\section{(Q5) Memory effect of the displacement term when $\gamma>0$ in (1.34) is not small}

The study in Subsection 1.2.3 was essentially carried out by a perturbation argument requiring that $\gamma$ be small. There is never any doubt that even when $\gamma$ is not small, the system will possess chaotic behavior. The memory effect of $\gamma w$ tends to cause a deformation of the invariant region, which we are unable to analyze so far.

We deem any successful study of the case when $\gamma>0$ is not small very desirable.

\section{(Q6) Coupled vibrating strings with a joint}

This problem is of the type considered in $[2,6]$.

The composite reflection relation for such a coupled structure is a $2 \times 2$ nonlinear matrix relation instead of the scalar map $G_{\eta} \circ F_{\alpha, \beta}$ or $G \circ F_{\nu}$ surveyed in this paper. This type of $2 \times 2$ nonlinear relation is not invertible, preventing the applicability of the Smale Horseshoe method.

New analytical methods are desirable to enable the treatment of 2-dimensional nonlinear mappings in order to handle (Q6). 


\section{References}

[1] Cartwright M and Littlewood J (1945) On nonlinear differential equations of the second order: I. The equation $\ddot{y}-k\left(1-y^{2}\right) \dot{y}+y=b \lambda k \cos (\lambda t+\alpha), k$ large. J London Math Soc 20:180-189

[2] Chen G, Hsu SB, and Zhou J (1996) Linear superposition of chaotic and orderly vibrations on two serially connected strings with a van der Pol joint. Int. J Bifurcation \& Chaos 6:1509-1527

[3] Chen G, Hsu SB, and Zhou J (1998) Chaotic vibrations of the one-dimensional wave equation subject to a self-excitation boundary condition, Part I. Transactions Amer Math Soc 350:4265-4311

[4] Chen G, Hsu SB, and Zhou J (1998) Ibid, Part II, energy injection, period doubling and homoclinic orbits. Int J Bifurcation \& Chaos 8:423-445

[5] Chen G, Hsu SB, and Zhou J (1998) Ibid, Part III, natural hysteresis memory effects. Int J Bifurcation \& Chaos 8:447-470

[6] Chen G, Hsu SB, and Zhou J (1998) Snapback repellers as a cause of chaotic vibration of the wave equation due to a van der Pol boundary condition and energy injection in the middle of the span. J Math Phys 39:6459-6489

[7] Chen G, Hsu SB, and Zhou J (2002) Nonisotropic spatiotemporal chaotic vibration of the wave equation due to mixing energy transport and a van der Pol boundary condition. Int J Bifurcation and Chaos 12:535-559

[8] Chen G, Lasiecka I, and Zhou J (eds) (2001) Unbounded growth of total variations of snapshots of the 1D linear wave equation due to the chaotic behavior of iterates of composite nonlinear boundary reflection relations, in Advances in Control of Nonlinear Distributed Parameter Systems. Marcel Dekker, New York, 15-42

[9] Chen G, Hsu SB, and Huang TW (2002) Analyzing the displacement terms memory effect to prove the chaotic vibration of the wave equation. Int J Bifurcation and Chaos 12:965-981

[10] Chen G, Huang T, and Huang Y (2003) Chaotic behavior of interval maps and total variations of iterates. To appear in Int J Bifurcation and Chaos

[11] Devaney RL (1989) An Introduction to Chaotic Dynamical Systems. Addison-Wesley, NY

[12] Guckenheimer J and Holmes P (1983) Nonlinear Oscillations, Dynamical Systems, and Bifurcation of Vector Fields. Springer-Verlag, NY

[13] Keener JP (1980) Chaotic behavior in piecewise continuous difference equations. Transactions Amer Math Soc 261:589-604

[14] Levi M (1981) Qualitative analysis of the periodically forced relaxation oscillations. Mem Amer Math Soc 214:1-147

[15] Li C and McLaughlin D (1994) Morse and Melnikov functions for NLS Pde's. Comm Math Phys 162:175-214

[16] Li C, McLaughlin D, Shatah J and Wiggins S (1996) Persistent homoclinic orbits for perturbed nonlinear Schrödinger equation. Comm Pure Appl Math XLIX:1175-1255

[17] Li C (1999) Smale horseshoe and symbolic dynamics in perturbed nonlinear Schrödinger equations. J Nonlinear Sci 9:363-415 
[18] Lorenz EN (1963) Deterministic non-periodic flow. J Atmos Sci 20:130-141

[19] Holmes P and Marsden J (1981) A partial differential equation with infinitely many periodic orbits: chaotic oscillators of a forced beam. Arch Rat Mech Anal 76:135-165

[20] Melnikov VK (1963) On the stability of the center for time periodic perturbations. Transactions Moscow Math Soc 12:1-57

[21] Russell DL (1974) Exact boundary value controllability theorems for wave and heat processes in star-complemented regions, in "Differential Games and Control Theory", Roxin, Liu and Sternberg, (eds) Marcel Dekker, NY

[22] Wiggins S (1990) Introduction to Applied Nonlinear Dynamical Systems and Chaos. Springer-Verlag, NY 